\title{
Componentes presentes en el aceite de fritura usado y determinantes previos a su conversión en biodiesel
}

\author{
Components present in the frying oil used and determinants previous to its \\ conversion into biodiesel
}

\author{
Herminia Sanaguano Salguero', Favian Bayas Morejón², Carlos Cabrera Carranza³
}

Recibido: Junio 2019 - Aprobado: Julio 2019

\begin{abstract}
RESUMEN
Los aceites residuales representan un problema para el medio ambiente, debido a que contaminan el agua produciendo una captación del oxígeno que impide la subsistencia de los organismos acuáticos. Esta investigación tuvo el objetivo de determinar los componentes presentes en aceite de fritura usado, procedente desde restaurantes en la ciudad de Guaranda, Bolívar-Ecuador previo a su conversión a Biodiesel. Se obtuvieron muestras de tres restaurantes de comidas rápidas los más populares de la ciudad en mención. Se realizaron análisis fisicoquímicos teniendo como punto de partida una filtración, los análisis realizados a los aceites fueron densidad, Acidez, humedad o pérdida por calentamiento e índice de refracción, y su comparación con parámetros normativos. Tras el análisis de densidad de los tres tipos de muestra se obtuvo una media entre 0,918 y 0,920 gr/ $\mathrm{mL}$, En acidez, se obtuvieron valores entre 3,006\% y 3,293\%. Los valores de humedad fueron de en una media de 0,099\%. En índice de refracción el valor medio fue de 1,459. Estos valores, se alejan de las normativas para un aceite puro para que se pueda seguir utilizando como alimento, no obstante, estos aceites residuales con las características que presentaron, han sido aprovechados para su conversión en biodiesel.
\end{abstract}

Palabras clave: Componentes; Aceit https://revistasinvestigacion.unmsm.edu.pe/index.php/iigeo/\$\$ $\$$ call $\$ \$$ /grid/article-galleys/article-galleygrid/add-galley?submissionld=17283 es de fritura; conversión; biodiesel.

\begin{abstract}
The residual oils represent a problem for the environment, because they contaminate the water producing an uptake of oxygen that prevents the subsistence of aquatic organisms. This investigation had the objective of determining the components present in the frying oil used from restaurants in the city of Guaranda, Bolívar - Ecuador prior to its conversion to Biodiesel. Samples were obtained from three fast food restaurants the most popular in the city in mention. Physicochemical analyzes were carried out with a filtration starting point, the analyzes performed on the oils were density, acidity, humidity or loss due to heating and refractive index, and their comparison with normative parameters. After the density analysis of the three sample types, an average between 0,918 and $0,920 \mathrm{gr} / \mathrm{mL}$ was obtained. In acidity, values between $3,006 \%$ and $3,293 \%$ were obtained. The humidity values were of an average of $0,099 \%$. In the refractive index, the mean value was 1,459 . These values, move away from the regulations for a pure oil so that it can continue to be used as food, however, these waste oils with the characteristics they presented, have been used for conversion into biodiesel.
\end{abstract}

\footnotetext{
1 Carrera de Ingeniería Agroindustrial. Facultad de Ciencias Agropecuarias Recursos Naturales y del Ambiente. Universidad Estatal de Bolívar. CP: 020150 E-mail: hsanaguano@ueb.edu.ec hsanaguano@ueb.edu.ec

2 Centro de Investigación y Desarrollo Biotecnológico. Facultad de Ciencias Agropecuarias Recursos Naturales y del Ambiente. Universidad Estatal de Bolívar, CP: 020150 E-mail: fbayas@ueb.edu.ec

3 Departamento de Ingeniería Geográfica. Facultad de Ingeniería Geológica, Minera, Metalúrgica y Geográfica,.CP: 15081 E-mail: ccabrerac@unmsm.edu.pe 
Keywords: Components; frying oils; conversion; biodiesel.

\section{INTRODUCCIÓN}

El rápido crecimiento de diferentes sectores de consumo de aceite en los últimos años se debe a la modificación de los hábitos alimentarios. Actualmente, la mayor parte de los aceites y grasas comestibles se consumen después de haber sido sometidos a elevadas temperaturas, en procesos como fritura y horneo, de manera que estos sufren procesos de degradación (Valencia et al. 2002).

Las grasas o aceites residuales hoy en día representan un problema para el medio ambiente, dado a que contaminan el agua produciendo una captación del oxígeno que impide la subsistencia de los organismos acuáticos. Además, las grasas y aceites interfieren con el intercambio de gases entre el agua y la atmósfera e impiden la penetración de la luz solar. En cuanto a la salud, el aceite recalentado da lugar a una sustancia llamada acroleína, que causa irritación gástrica, también se generan compuestos aromáticos derivados del antraceno, (benzopireno y benzoantraceno), ambos agentes cancerígenos (Chinchilla et al. 2016).

El sector del transporte consume un $30 \%$ de toda la energía utilizada en el mundo. Esta cifra se eleva en la Unión Europea al 32\% y en España al 39\%. Actualmente, a nivel global se consumen diariamente 83,7 millones de barriles de petróleo y las estimaciones indican que esta cifra aumentará hasta llegar a los 112 millones de barriles por día en el 2020. Por ende, la escasez de petróleo está asegurada, por lo que encontrar y desarrollar soluciones alternativas no sólo es una cuestión ambiental, sino también una necesidad energética de futuro (Pardal, 2012).

El Protocolo de Kioto ha planteado exigencias en cuanto a los límites de emisiones causadas por el uso de combustibles fósiles. Según las proyecciones de emisiones de dióxido de carbono (CO2), para el año 2025 estas aumentarán entre 415 y 421 ppm. Por esta razón, es necesario buscar alternativas de combustibles, y es ahí donde aparece el biodiesel, dado a que el biodiesel genera menores emisiones de gases. La materia prima, para la obtención de este combustible es el aceite vegetal y porque no aprovecharlo del ya utilizado (Bocanegra et al. 2015).

El biodiesel es una de las mejores fuentes disponibles para cumplir esta demanda de energía en el mundo. Además, el hombre está obligado a contribuir con soluciones a la problemática ambiental, por esto los científicos han puesto las miradas en los biocombustibles y en especial en el biodiesel (Medina et al. 2015).

\section{MÉTODOS}

La presente investigación se llevó acabo en el laboratorio general de la Universidad Estatal de Bolívar, para lo cual se recolectaron muestras de tres restaurantes de comidas rápidas más populares de la ciudad de Guaranda, Ecuador (RP, FR y CA), Tras la recolección los aceites fueron transportados al laboratorio para su respectivo análisis.

Los aceites de fritura utilizados para elaborar biodiesel requieren de un pretratamiento, debido especialmente a que existen sólidos en suspensión por lo que se procede a una filtración en caliente (Bocanegra et al. 2015).

\subsection{Filtración en caliente}

La filtración en caliente puede realizarse por gravedad o por vacío. En el presente estudio se desarrolló por vació, para lo cual se utilizó un embudo Büchner para muestras voluminosas. Entre el embudo y kitazato se colocó un tapón de goma perforado, perfectamente adaptado a la boca del kitazato. La succión se realizó por la conexión lateral que presenta el kitazato, y el vació se consiguió utilizando una bomba mecánica. Sobre la placa perforada del embudo Büchner se colocó un papel filtro circular. La temperatura de filtración fue a $60{ }^{\circ} \mathrm{C}$ de acuerdo con lo descrito por (Lamarque y Zygadlo, 2008).

La filtración en caliente permite remover sustancias carbonosas producidas a partir de material orgánico quemado, restos de comida y otros sólidos infusibles (Bocanegra et al. 2015).

Los análisis realizados se basaron en las metodologías establecidas por las normas INEN (Instituto Nacional Ecuatoriano de Normalización) específicas para cada propiedad del aceite decolorado de palma como se resume en la Tabla 1.

Tabla 1. Especificación del aceite comestible de palma NTE INEN 2421

\begin{tabular}{lcccc}
\hline Propiedades & Unidad & MIN & MAX & Método de ensayo \\
\hline Densidad relativa $\left(25 / 25^{\circ} \mathrm{C}\right)$ & $\mathrm{gr} / \mathrm{ml}$ & 0,8957 & 0,91 & NTE INEN 35 \\
Humedad y materia volátil & $\%$ & - & 0,05 & NTE INEN 39 \\
Acidez (como ácido oleico) & $\%$ & - & 0,1 & NTE INEN 38 \\
Índice de refracción a $25^{\circ} \mathrm{C}$ & - & 1,454 & 1,456 & NTE INEN 42 \\
\hline
\end{tabular}

Fuente: Sanaguano 2017

\subsection{Densidad del aceite de fritura usado}

Densidad, este análisis se realizó de acuerdo con la Norma Técnica Ecuatoriana (NTE INEN) 2421 para grasas y aceites comestibles, el método de ensayo fue conforme la norma NTE INEN 35, mediante el uso de un picnómetro (Gay-Lussac, de $50 \mathrm{~cm}^{3}$ ) para determinar la densidad relativa a $25 / 25{ }^{\circ} \mathrm{C}$ de las grasas y aceites vegetales o animales. Densidad relativa a $25 / 25^{\circ} \mathrm{C},(25)$. Es la relación entre la masa de un volumen dado de una sustancia a $25^{\circ} \mathrm{C}$ y la masa de un volumen igual de agua a $25^{\circ} \mathrm{C}$.

\section{Cálculos}

Para los aceites y grasas líquidas a $25^{\circ} \mathrm{C}$, la densidad relativa a $25 / 25^{\circ} \mathrm{C}$ se calcula mediante la ecuación siguiente:

$$
\mathrm{d}_{25}=\frac{\mathrm{m}_{2}-\mathrm{m}}{\mathrm{m}_{1}-\mathrm{m}}
$$

Dónde: $\mathrm{d}_{25}=$ densidad relativa a $25 / 25^{\circ} \mathrm{C} ; \mathrm{m}=$ masa del picnómetro vacío, en $\mathrm{g} ; \mathrm{m}_{1}=$ masa del picnómetro con agua destilada, en $\mathrm{g} ; \mathrm{m}_{2}=$ masa del picnómetro con muestra, en $g$.

\subsection{Acidez del aceite de fritura usado}


Este análisis fue de acuerdo con el método NTE INEN 038. En una grasa o aceite, el contenido de ácidos grasos libres, expresado convencionalmente como gramos de ácido oleico, laúrico o erúcico por cada $100 \mathrm{~g}$ de sustancia. Índice de acidez, es el número de miligramos de hidróxido de potasio requeridos para neutralizar los ácidos grasos libres contenidos en 1 gramo de grasa o aceite (Rivera et al. 2014).

\section{Cálculos}

La acidez se calcula mediante la ecuación siguiente:

$$
\mathrm{A}=\frac{\mathrm{M} * \mathrm{~V} * \mathrm{~N}}{10 * \mathrm{~m}}
$$

Dónde: $\mathrm{A}=$ acidez del producto, en porcentaje de masa; $\mathrm{M}=$ masa molecular del ácido usado para expresar el resultado (ácido oleico $282 \mathrm{~g} / \mathrm{mol}$ ); $\mathrm{V}=$ volumen de la solución de hidróxido de sodio o de potasio empleado en la titulación, en $\mathrm{cm}^{3} ; \mathrm{N}=$ normalidad de la solución de hidróxido de sodio o de potasio; $\mathrm{m}=$ masa de la muestra analizada, en gramos.

\subsection{Humedad o pérdida por calentamiento}

En este trabajo se utilizó el método de la plancha de calentamiento que es aplicable a todos los aceites y grasas vegetales o animales, de acuerdo con el método NTE INEN 39.

Para lo cual el aceite se calienta a $103{ }^{\circ} \mathrm{C}$ hasta eliminar completamente la humedad y las materias volátiles.

\section{Preparación}

Si la muestra es líquida y presenta aspecto claro y sin sedimento, homogeneizarla invirtiendo varias veces el recipiente que la contiene. Si la muestra es líquida y presenta aspecto turbio o con sedimento, invertir varias veces el recipiente que la contiene, hasta que el sedimento se haya separado completamente de las paredes del recipiente y se haya distribuido uniformemente en la masa del aceite.

\section{Cálculos}

La pérdida por calentamiento se calcula mediante la ecuación siguiente:

$$
\mathrm{P}=\frac{\mathrm{m}_{1}-\mathrm{m} 2}{\mathrm{~m}_{1}-\mathrm{m}} \times 100
$$

$\mathrm{P}=$ perdida por calentamiento, en porcentaje de masa

$\mathrm{m}=$ masa de la capsula con el termómetro, en gramos $\mathrm{m} 1=$ masa de la capsula con el termómetro y la muestra, antes del calentamiento, en gramos.

$\mathrm{m} 2=$ masa de la capsula con el termómetro y la muestra, después del calentamiento, en gramos.

La diferencia entre los resultados de una determinación efectuada por duplicado no debe exceder del $0,05 \%$; en caso contrario debe repetirse la determinación. (Norma INEN 039).

\section{5. Índice de refracción}

El índice de refracción es una de las constantes físicas más importantes para identificar un compuesto; además los refractómetros permiten determinen el índice con gran precisión, dependiendo este valor de la longitud de onda utilizada y de la temperatura. Como temperatura normal se suele tomar $20{ }^{\circ} \mathrm{C}$ y la longitud de onda standard es la correspondiente a la línea D del espectro del yodo (589 nm) (Solano y Pérez, 1991).

El índice de refracción del agua es $\mathbf{n}_{\mathrm{D}}^{20}=1,3333$

La medida del índice de refracción es muy útil para el estudio de los líquidos. Su relación con la densidad permite en ocasiones facilitar su análisis cuantitativo.

En este estudio se utilizó un refractómetro (Abbe).

\section{RESULTADOS}

\subsection{Densidad de aceite de fritura}

La condición física del aceite de fritura que se recolectó de los tres restaurantes presentó un aspecto heterogéneo en su textura. El color de aceite fue café obscuro, olor muy fuerte y con poca cantidad de sólidos, denso, poco viscoso y luego de tres meses de almacenamiento de los aceites, los recipientes generalmente presentaron dos fases, una fase (líquida) menos densa y la otra más densa (sólida). La fase densa de un color café claro, olor fuerte y textura muy pastosa que se encontraba pegada a las paredes del recipiente plástico. De tal modo que, se requirió de un pretratamiento, mismo que consistió en separar la parte sólida y los residuos de alimentos mediante una filtración.

Los resultados de estas mediciones se presentan en la tabla 2, los promedios de densidad de las tres muestras de aceites presentaron valores entre 0,918 a $0,920 \mathrm{gr} / \mathrm{ml}$ los mismos que sobrepasan el límite máximo de la Norma INEN 35 establecida para aceites comestibles, lo cual es un indicativo de que estos aceites presentan una mayor densidad de un aceite sin usar, esto se debe a su saturación y por ende al mayor tiempo de calentamiento que ha

\begin{tabular}{|c|c|c|c|c|c|c|c|}
\hline \multicolumn{4}{|c|}{$\begin{array}{c}\text { Densidad } \\
\left(50^{\circ} \mathrm{C} / \text { agua a } 20^{\circ} \mathrm{C}\right)(\mathrm{gr} / \mathrm{ml})\end{array}$} & \multirow{2}{*}{$\begin{array}{c}\text { Promedio } \\
0,920\end{array}$} & \multirow{2}{*}{$\begin{array}{c}\text { Mínimo } \\
0,8957\end{array}$} & \multirow{2}{*}{$\begin{array}{c}\text { Máximo } \\
0,910\end{array}$} & \multirow{2}{*}{$\begin{array}{c}\begin{array}{c}\text { Método } \\
\text { de Ensayo }\end{array} \\
\text { NTE INEN } 35\end{array}$} \\
\hline Muestra 1 & 0,920 & 0,917 & 0,923 & & & & \\
\hline Muestra 2 & 0,918 & 0,921 & 0,922 & 0,920 & 0,8957 & 0,910 & NTE INEN 35 \\
\hline Muestra 3 & 0,916 & 0,918 & 0,920 & 0,918 & 0,8957 & 0,910 & NTE INEN 35 \\
\hline
\end{tabular}

Tabla 2. Densidad de tres muestras de aceite de fritura usado

Fuente: Sanaguano 2017 
pasado el aceite. Los valores obtenidos de estos análisis son aproximados a los datos presentados por (Murcia et al. 2013), donde reporta $0,911 \mathrm{gr} / \mathrm{ml}$ para el aceite de cocina (desecho). Mientras que, Medina et al. (2015) obtuvieron valores de $0,924 \mathrm{gr} / \mathrm{ml}$ que es un dato mayor al de los aceites analizados en este estudio.

\section{2. Índice de acidez del aceite de fritura}

Los valores de los análisis de acidez de las muestras de los aceites de fritura se presentan en la Tabla 3.

Tabla 3. Datos de acidez del aceite de fritura

\begin{tabular}{ccccccc}
\hline Muestra & \multicolumn{3}{c}{ Acidez (como ácido oleico) } & \multicolumn{3}{c}{ Método de ensayo } \\
& \multicolumn{1}{c}{} & & & \multicolumn{3}{c}{ NTE INEN 38 } \\
\hline & & $(\%)$ & & Promedio & Mínimo & Máximo \\
\hline 1 & 3,198 & 3,221 & 3,312 & 3,243 & - & 0,10 \\
2 & 3,189 & 3,195 & 3,19 & 3,191 & - & 0,10 \\
3 & 3,02 & 3,010 & 2,978 & 3,006 & - & 0,10 \\
\hline
\end{tabular}

Fuente: Sanaguano 2017

La Norma NTE INEN 38 establece un máximo de $0,1 \%$ de acidez para los aceites comestibles y al comparar con los datos obtenidos se determinó que todos los valores superan el valor máximo de la Norma, estos resultados son un indicador de que el aceite pasó por una etapa de calentamiento prolongado. Estos valores de acidez se deben probablemente a que a mayor tiempo de fritura se produce una liberación de ácidos grasos desde los triglicéridos que forman los distintos aceites, lo cual puede favorecer la formación de humo y/o de sabores indeseables (rancidez hidrolítica) manifiesta (Ayala Ramirez, 2011).

Murcia et al. (2013), manifiestan que, la presencia de calor y agua acelera la hidrólisis de triglicéridos y por tanto aumenta el contenido de ácidos grasos libres. Los valores de acidez de la tabla 3 , presentan valores bajos comparados con otros aceites de fritura que han sido estudiados por Murcia et al. (2013) que reporta valores de 9,193 (\% m/m ácido oleico) para el aceite de cocina (desecho) y de 1,87 para los aceites de cocina usados.

Bulla et al. (2014), en su trabajo realizado sobre el análisis de acidez de aceites de fritura usados determinó valores de 7,65 lo que se deduce que los índices de acidez de las tres muestras de aceites analizadas en este estudio presentan valores menores comparados con los datos de los otros autores, por tanto, los aceites analizados no poseen mucha acidez.

\subsection{Humedad del aceite de fritura}

El análisis de humedad que se realizó en las muestras de aceite de fritura usado se determinó de acuerdo con la Norma NTE INEN 39, los resultados se presentan en la Tabla 4.

En base a los datos de la tabla 4, se observa que sobrepasan el valor máximo permitido por la norma NTE INEN 39 que establece que el valor máximo de humedad y material volátil de los aceites comestible es de $0,05 \%$. En el trabajo realizado por Murcia et al. (2013) sobre esta característica reportan valores de humedad en los aceites de fritura con porcentajes de 0,1046 mayor a los obtenidos en este trabajo.

\section{4. Índice de refracción del aceite de fritura}

El análisis del índice de refracción se realiza en base a la norma NTE INEN 42 y los resultados se presentan en la Tabla 5. Esta propiedad física de los aceites comestibles es característico dentro de ciertos límites para cada aceite por lo que es un indicador de la pureza del aceite. Este valor está relacionado con el grado de saturación, con la razón cis/trans de los dobles enlaces y puede estar influenciando por el daño que sufre el aceite tras la oxidación expone (Soto Vásquez, 2011), y a la vez determina índice de refracción de algunos aceites: aceite de maíz 1,465-1,468, aceite de semilla de mostaza 1,461-1,469, aceite de palma 1,454-1,456 y aceite de almendra de palma 1,448-1452.

Murcia et al. (2013), obtuvieron valores de índice de refracción de 1,4605 a $50{ }^{\circ} \mathrm{C}$ de aceite de cocina de desecho,

Tabla 4. Análisis de humedad del aceite de fritura usado

\begin{tabular}{lccccccc}
\hline \multicolumn{2}{l}{ Humedad y materia volátil \% en masa } & & Promedio & Mínimo & Máximo & Método de ensayo \\
\hline Muestra 1 & 0,109 & 0,099 & 0,101 & 0,103 & - & 0,05 & NTE INEN 39 \\
Muestra 2 & 0,099 & 0,095 & 0,104 & 0,099 & - & 0,05 & NTE INEN 39 \\
Muestra 3 & 0,089 & 0,095 & 0,090 & 0,091 & - & 0,05 & NTE INEN 39 \\
\hline
\end{tabular}

Fuente: Sanaguano 2017

Tabla 5. Índice de refracción del aceite de fritura

\begin{tabular}{lccccccc}
\hline \multicolumn{2}{l}{ Índice de refracción a $25^{\circ} \mathbf{C}$} & & & Promedio & Mínimo & Máximo & Método de ensayo \\
\hline Muestra 1 & 1,460 & 1,462 & 1,460 & 1,461 & 1,454 & 1,456 & NTE INEN 42 \\
Muestra 2 & 1,459 & 1,458 & 1,458 & 1,458 & 1,454 & 1,456 & NTE INEN 42 \\
Muestra 3 & 1,457 & 1,458 & 1,457 & 1,457 & 1,454 & 1,456 & NTE INEN 42 \\
\hline
\end{tabular}

Fuente: Sanaguano 2017 
Tabla 6. Análisis físico químico de biodiesel obtenido a temperatura sub crítica

\begin{tabular}{lccccc}
\hline Análisis & Muestra 1 & Muestra 2 & Muestra 3 & Rango & Método \\
\hline Punto de inflamación ${ }^{\circ} \mathrm{C}$ & 183,2 & 181,2 & 185,1 & $86,6-224,1$ & PNE/DPEC/P/ \\
Índice de cetano calculado & 46 & 45 & 45 & $40-60$ & ASTM D-92 \\
PNE/DPEC/P/ & ASTM D287 \\
Densidad ${ }^{\circ} \mathrm{API}$ a 60 $60^{\circ} \mathrm{F}$ & 28,5 & 27,9 & 28,3 & $9,3-99,4$ & PNE/DPEC/P/ \\
$\begin{array}{l}\text { Viscosidad cinemática } \\
\text { a 40 }\end{array}$ & 4,75 & 5,33 & 5,09 & $1,15-4,66$ & ASTM D287 \\
\hline
\end{tabular}

Muestra $1(9: 1-1 \%)$ a $180^{\circ} \mathrm{C}$; Muestra $2(9: 1-0,5 \%)$ a $180^{\circ} \mathrm{C}$; Muestra $3(6: 1-0,5 \%)$ a $180^{\circ} \mathrm{C}$.

Fuente: Sanaguano 2017

comparado con los valores promedios de este trabajo a la temperatura de $25{ }^{\circ} \mathrm{C}$ están cercanos. Comparados con la Norma NTE INEN 42 sobrepasan el valor máximo para aceites comestibles que es de 1,456. Finalmente se concluye que, los valores de índice de refracción de la Tabla 5, expresan que las tres muestras de aceites no son puros y que tiene un grado de saturación.

Con las características previamente indicadas del aceite de frituras se procedió a la conversión a biodiesel, mediante una reacción de transesterificación, con metanol en condiciones subcrítica e hidróxido de sodio como catalizador, logrando obtener un biodiesel con las características que se resumen en la Tabla 6.

\section{CONCLUSIONES}

Los aceites de fritura residual analizados en esta investigación, se determinan los siguientes componentes: densidad, acidez, humedad e índice de refracción, los valores estimados de estos análisis son superiores a los valores máximos que establece la Norma INEN para los aceites vegetales, por tanto, se considera que estos aceites no son aptos para el consumo como alimento.

Los aceites de fritura residuales que ya no son útiles como alimento, porque, no cumplen con las Normas INEN, $\mathrm{y}$ a este residuo alimenticio que no tiene otra utilidad, ha sido aprovechado para su conversión en biodiesel. La densidad del aceite de fritura fue de $0,92 \mathrm{gr} / \mathrm{mL}$ y luego de la conversión a biodiesel su densidad fue de $0,88 \mathrm{gr} /$ $\mathrm{mL}$ que de acuerdo al ASTM - D1298 el valor de densidad permitido es de entre $0,85-0,90 \mathrm{~g} / \mathrm{cm} 3$. Otras propiedades físicas analizadas del biodiesel son: Punto de inflamación 183,2 ${ }^{\circ} \mathrm{C}$; Índice de cetano calculado 46; Viscosidad cinemática a $40{ }^{\circ} \mathrm{C} 4,75$ (cSt), estos valores están dentro de los rangos de las normas ASTM.

\section{AGRADECIMIENTOS}

A la Universidad Estatal de Bolívar- Ecuador, al Laboratorio de la Facultad de Ciencias Agropecuarias, y al Dr. Carlos Cabrera por su asesoramiento y guía para el mejor desarrollo del presente artículo de investigación.

\section{REFERENCIAS BIBLIOGRAFICAS}

Ayala Ramírez, M.J. (2011). Evaluación de la calidad del aceite de mezclas vegetales utilizado en doce frituras sucesivas empleado para freír plátano hartón verde. (Tesis de grado Pregrado). Facultad de Ciencias, Pontificia Universidad Javeriana, Bogotá. Recuperado de http://hdl.handle. net/10554/8796

Bocanegra, J., Malagón, D., y López, L. (2015). Obtención de biodiesel a partir de aceite usado de cocina por transesterificación. Ingeniería Y Universidad, 19(1), 155-172. https://doi.org/10.11144/Javeriana.iyu19-1.odba

Bulla Pereira, E. A., Sierra, F. E., \& Guerrero, C. A. (2014). Biodiesel Production Using Waste Cooking Oil and Ethanol for Alkaline Catalysis. Ingeniería Solidaria, 10(17), 61-69. https://doi.org/10.16925/in.v9i17.806

Chinchilla, N., Sagot, P., y Villalobos, G. (12 de Agosto de 2016). Educación nutricional y ambiental en el manejo de grasas residuales. Costarricense de Salud Pública, 25, 20 - 27. Recuperado de https://www.scielo.sa.cr/pdf/rcsp/ v25n2/1409-1429-rcsp-25-02-00020.pdf

Lamarque, A., Maestri, D., Labuckas, D., Zygadlo, J. y Torres, M. (2008). Fundamentos teórico-prácticos de química orgánica. Córdoba, Argentina: Encuentro Grupo Editor. Recuperado de http://bit.ly/2EtJ6ob

Medina Villadiego, Mauricio; Ospino Roa, Yesid y Tejeda Benítez, Lesly (2015). Esterificación y transesterificación de aceites residuales para obtener biodiesel. Revista Luna Azul, núm. 40, enero-junio, 2015, pp. 25-34. Universidad de Caldas, Manizales, Colombia. Recuperado de https://www. redalyc.org/pdf/3217/321733015003.pdf

Murcia, B.; Chaves, L.; Rodríguez-Pérez, W., Andredy, E. y Alvarado, E. (2013) Caracterización de Biodiesel Obtenido de Aceites Residual de cocina. Rev. Colomb. Biotecnol. Vol. XV No. 1 Julio 2013 61-70. Recuperado de https://dialnet. unirioja.es/descarga/articulo/4776363.pdf

NTE INEN 2421 (Norma Técnica Ecuatoriana-Instituto 
Ecuatoriano de Normalización) (2009): Grasas y aceites Aceites comestibles. Aceite de palma híbrida (OG) "OLECO". Recuperado de http://www.fao.org/faolex/ results/details/en/c/LEX-FAOC092416

Pardal, A. (2012). Obtención de biodiesel por transesterificación de aceites vegetales: nuevos métodos de sinteses. (Tese de Doutoramento não publicada). Facultat de Ciencias, Universidad de Extremadura (Espanha). Recuperado de http://hdl.handle.net/20.500.12207/738

Rivera, Yezabel; Gutiérrez, Carlos; Gómez, Rubén; Matute, María y Izaguirre, César (2014). Cuantificación del deterioro de aceites vegetales usados en procesos de frituras en establecimientos ubicados en el Municipio Libertador del Estado Mérida. Ciencia e Ingeniería, vol. 35, núm. 3, agosto-noviembre, 2014, pp. 157-164. Universidad de los Andes Mérida, Venezuela. ISSN: 1316-7081. Recuperado de https://www.redalyc.org/ pdf/5075/507550626005.pdf
Solano, E.; Perez, E.; y Alonso, T. (1991) Prácticas de laboratorio de Química Orgánica. Murcia: EDITUM. Recuperado de http://bit.ly/2CPKj81

Soto Vásquez, M. (2011). Control de Calidad de Aceites Vegetales. Docente investigador Universidad Nacional de Trujillo. Investigadora Concytec. Recuperado de https:// es.slideshare.net/maryluz/control-de-calidad-de-aceitesvegetales-por-qf-maril-roxana-soto-vsquez

Valencia, E.; Valenzuela, E.; Nuñez, P.; y Barrera, D. (2002). Estudio de algunas características fisicoquimicas de algunos aceites comerciales sometidos a procesos intermitentes de frituras de papas. Información Tecnológica Vol. $13 \mathrm{~N}^{\circ} 4$, pp.19-24. Recuperado de http://bit.ly/2QpqoFk 